\title{
The nano-mechanics and magnetic properties of high moment synthetic antiferromagnetic particles
}

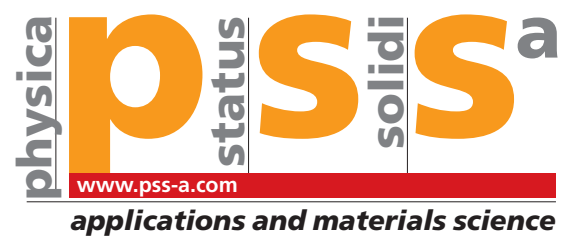

\author{
Michael Forrester ${ }^{*}$ and Feodor Kusmartsev \\ Department of Physics, Loughborough University, Epinal Way, Loughborough LE11 3TU, United Kingdom
}

Received 12 July 2013, revised 31 October 2013, accepted 11 December 2013

Published online 21 January 2014

Keywords anisotropy, antiferromagnetic nanoparticles, magnetization, nano-mechanics

*Corresponding author: e-mail d.m.forrester@lboro.ac.uk, Phone: +44 (0) 1509228208

With nanomagnets increasingly being used and proposed as functional units for in vivo applications, it is vital to understand how to optimize their structure, geometry, and size, and their responses to electromagnetic stimulation. Herein, we predicate how to do so for synthetic antiferromagnetic structures that are subjected to external magnetic control. Because the structures are on the scale of biological entities, interactions with cells and molecular constituents can be extreme and careful design must be undertaken to avoid detrimental effects. Thus, the magnetic responses of multilayers, as demonstrated in experiments by Koh et al. [e.g., Hu et al., Adv. Mater. 20, 1479 (2008) and Koh et al., J. Appl. Phys. 107, 09B522 (2010)], are understood using a fully dynamical investigation based on LandauLifshitz-Gilbert equations. We find that during the fabrication of the structures the axial positions of the nanomagnets become offset from each other, leading to the characteristic magnetic hysteresis shapes witnessed. We then find the magnetic nanomechanical forces generated by such structures.

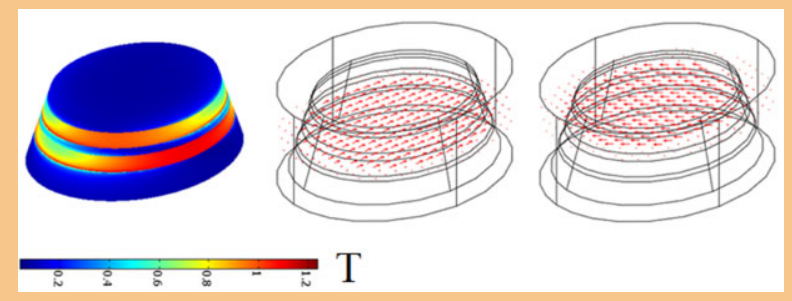

The conical synthetic antiferromagnetic nanoparticles with two magnetic layers - Left: the magnetic flux density is shown on the surface of the structure in a magnetic flux density of $B=0.08 \mathrm{~T}$. Middle and right: orientations of the magnetic moments of the two magnetic layers.
1 Introduction The manufacture of synthetic antiferromagnetic (SAF) nanoparticles for biomedical applications is of high importance because of their outstanding magnetic properties [1]. These SAF structures are designed to possess very little remanence in the absence of an applied magnetic field. They also have high-magnetic moments $\left(\approx 850 \mathrm{emu} \mathrm{cm}^{-3}\right)$ as compared to superparamagnetic iron oxide nanoparticles [2]. In this work, we examine the conical structure of the SAF's and determine their dynamical magnetic characteristics. It is found that during the fabrication of the nanoparticles that the magnetic disks become slightly elongated with their longest axes offset from one another. Making use of Landau-Lifshitz-Gilbert (LLG) equations we reproduce the experimental hysteresis curves of Koh et al. [2] for SAF nanoparticles. Further to this, we are then able to identify how to tailor the magnetic properties of the SAF during fabrication by exploiting the elongation of the nanoparticles and thereby making elliptical cone structures. The magnetic forces on the SAF structure are examined and manipulated based upon the effective magnetic permeability, saturation magnetization, and also the geometry. We find that in principle femto to piconewton forces can be generated in moderate magnetic fields. Tailoring the nano-mechanics so as to exert specific magnetic torques is important for investigating biological structures and for the treatment of disease. For example, tensions on the order of $100 \mathrm{fN}$ can slow or prevent the formation of DNA loops - providing a means to regulate gene expression [3, 4]. Or larger piconewton forces in the intracellular environment can be used to initiate apoptosis in cancer cells [5].

\section{Synthetic antiferromagnetic nanoparticles}

2.1 Dynamical evolution We investigate the SAF's that are typically composed of two or more ferromagnetic 
layers made of $\mathrm{Co}_{90} \mathrm{Fe}_{10}$, for example [2]. Between these magnetic compounds there are nonmagnetic spacer layers (e.g., made of ruthenium). Depending upon the thicknesses of the interlayers, the nanomagnets are either coupled through exchange or dipole-dipole interactions.

Figure 1 shows the conical structure of the SAF. In each $\mathrm{SAF}$, there are at least two magnetic layers. We show that each layer is skewed in relation to the other, giving rise to the shape of the hysteresis profile that is seen in Fig. 1. Our finding of an elliptical geometry is derived within the nominal values for the dimensions stated in the experimental paper by Koh et al. [2]. In order to describe the magnetic energy of the SAF structure with $N$ magnetic layers, and its magnetic response, we write the energy equation that contains coupling strength $(J)$, anisotropy constants $(K)$, magnetization $(M)$, and an applied magnetic field $\left(H_{\mathrm{a}}\right)$

$$
\begin{aligned}
E= & -\mu_{0} V \sum_{\langle i j\rangle}^{N} J \mathbf{M}_{i} \cdot \mathbf{M}_{j}+K_{x, i} M_{x, i}^{2} \\
& +K_{y, i} M_{y, i}^{2}+K_{z, i} M_{z, i}^{2}+\mathbf{H}_{\mathrm{a}} \cdot \mathbf{M}_{i} .
\end{aligned}
$$

The comparison of theory to the experimental results [2] before and after ion milling is conducted by utilizing the LLG equation:

$$
\frac{\partial \mathbf{M}_{i}}{\gamma \partial t}=\left[\mathbf{M}_{i} \times \mathbf{H}_{\mathrm{eff}, i}\right]-\frac{\alpha}{M_{\mathrm{S}}}\left[\mathbf{M}_{i} \times\left[\mathbf{M}_{i} \times \mathbf{H}_{\mathrm{eff}, i}\right]\right],
$$

where the saturation magnetization is denoted as $M_{\mathrm{S}}$ and the gyromagnetic ratio by $\gamma$. The last term of the LLG equation introduces damping and the Gilbert damping parameter, $\alpha$. The effective field is given by

$$
\mathbf{H}_{\mathrm{eff}, i}=-\frac{1}{\mu_{0} V} \frac{\partial E}{\partial \mathbf{M}_{i}} .
$$
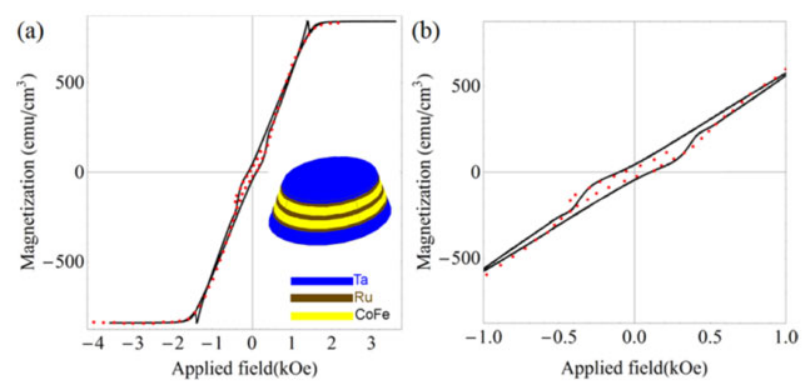

Figure 1 (a) The magnetic hysteresis loops of a sample of $\mathrm{Co}_{90} \mathrm{Fe}_{10}(6) / \mathrm{Ru}(3) / \mathrm{Co}_{90} \mathrm{Fe}_{10}(5.4)$. The solid black lines are derived from the LLG equations and compared to the experimental data (red dots) of Koh et al. [2]. The SAF structure is that of a skewed elliptical cone. (b) Magnification of the hysteresis loop between an applied field of $\pm 1 \mathrm{kOe}$. The bottom magnet has cross-sectional dimensions $l_{x}=90 \mathrm{~nm}$ and $l_{y}=83 \mathrm{~nm}$. For the top magnet, $l_{x}=73 \mathrm{~nm}$ and $l_{y}=82 \mathrm{~nm}$. For the bottom nanomagnet, $\left\{K_{x, 1}\right.$, $\left.K_{y, 1}, K_{z, 1}\right\}=\{0.0740,0.0830,0.843\}$ are the anisotropy parameters whereas for the top one $\left\{K_{x, 2}, K_{y, 2}, K_{z, 2}\right\}=\{0.0852,0.0728,0.842\}$.
Each nanomagnet in the SAF has volume $V=(\pi / 4) l_{x} l_{y} l_{z}$, where $l_{x, y, z}$ are the lengths along the $x, y$, and $z$ axes. The cross-section in the $x-y$ plane is elliptical and each nanomagnet is approximately an elliptical cylinder. The longest axis of one nanomagnet is offset from that of the other. In a SAF structure with perfectly circular disks, there is no hysteresis and the remanence and coercivity are zero (see Supporting Information, online at www.pss-a.com). In order to reproduce the experimental results, a small misalignment of the easy axes of the ferromagnets in the SAF is assumed. In the example considered in Fig. 1, the best fits are for a bottom nanomagnet of dimensions $l_{x}=90 \mathrm{~nm}, l_{y}=83 \mathrm{~nm}, l_{z}=6 \mathrm{~nm}$; and a top one of dimensions $l_{x}=73 \mathrm{~nm}, l_{y}=82 \mathrm{~nm}$, $l_{z}=5.4 \mathrm{~nm}$. These values lie within the nominal values specified for the SAF's [2]. An external magnetic field is applied in the $x-y$ plane at $7^{\circ}$ to the easy-axis of the bottom nanomagnet. It is found using magneto-static simulations [6] that the coupling energy between the nanomagnets is approximately $0.3 \mathrm{erg} \mathrm{cm}^{-2}$. This coupling reduces in size after ion milling to about $0.1 \mathrm{erg} \mathrm{cm}^{-2}$, and this is the
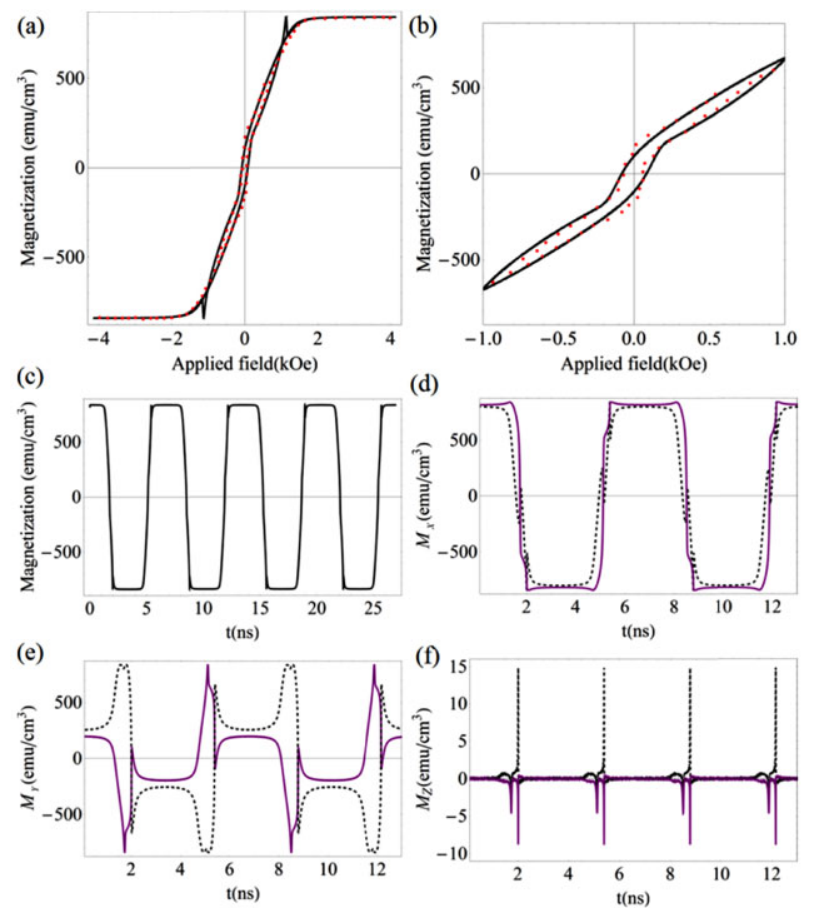

Figure 2 After ion milling the SAF magnetic character changes. The bottom magnet now has cross-sectional dimensions $l_{x}=85 \mathrm{~nm}$ and $l_{y}=76 \mathrm{~nm}$. For the top magnet, $l_{x}=70 \mathrm{~nm}$ and $l_{y}=75 \mathrm{~nm}$. The nominally stated thicknesses may also change and for the bottom nanomagnet $l_{z}=6.45 \mathrm{~nm}$ and for the top one $l_{z}=5.8 \mathrm{~nm}$. The hysteresis loop is shown in (a) and (b). In (c), the average magnetization is shown as a function of time. In (d-f), the time evolution of the components of the magnetization for each nanomagnet are shown (purple solid lines for the first magnet and dashed black lines for the second). Here, the anisotropy parameters for the bottom nano-magnetic layer are $\left\{K_{x, 1}, K_{y, 1}, K_{z, 1}\right\}$ $=\{0.0806,0.0944,0.825\}$, while for the top layer $\left\{K_{x, 2}, K_{y, 2}\right.$, $\left.K_{z, 2}\right\}=\{0.0855,0.0778,0.8367\}$. 
coupling strength for the system, described in Fig. 2. The anisotropy is related to the size of the structure and any geometrical deviations affect it. Shape anisotropy is present with its largest component perpendicular to the plane of the SAF structure. The magnetic particles can be described as elliptical cylinders with demagnetization factors [7]

$$
\begin{aligned}
K_{Y}= & \frac{8 \rho}{3 \pi^{2} \tau\left(1-\rho^{2}\right)}\left[\rho^{2} L\left(1-\rho^{2}\right)-\sigma\left(1-\rho^{2}\right)\right] \\
& +\frac{2 \rho^{2}}{\pi} \int_{0}^{\pi / 2} \mathrm{~d} \phi \frac{\cos ^{2} \phi}{g(\rho, \phi)} F\left[-\frac{\rho^{2}}{\tau^{2} g(\rho, \phi)}\right]
\end{aligned}
$$

and

$$
\begin{aligned}
K_{Z}= & +\frac{8 \rho}{3 \pi^{2} \tau} L\left(1-\rho^{2}\right) \\
& -\frac{2}{\pi} \int_{0}^{\pi / 2} \mathrm{~d} \phi F\left[-\frac{\rho^{2}}{\tau^{2} g(\rho, \phi)}\right],
\end{aligned}
$$

where $L$ and $\sigma$ are complete elliptic integrals of the first and second kinds, respectively. The ratios $\rho=l_{x} / l_{y}$ and $\tau=l_{z} / 2 l_{x}$ contain the lengths associated with the elliptic cylinder. The hyper-geometric function used is $F(x)={ }_{2} F_{1}[-1 / 2,1 / 2 ; 2, x]$, $g(\rho, \phi)=\sin ^{2} \phi+\rho^{2} \cos ^{2} \phi$.

These equations, (4) and (5), allow us to estimate the anisotropy demagnetization factors, which are related to one another by $K_{x}+K_{y}+K_{z}=1$. Stress on the SAF structure results in an additional strain anisotropy, $K \rightarrow K+\delta K$, when the interfaces between the two magnetic materials and their interlayer are stretched to match one another. Additionally, $\delta K$ may be due to deposition defects or material imperfections.

In the work of Koh et al. [2], it was found that the hysteresis profile changed when the SAF structures were immersed in water. There was an increase in energy loss that was demonstrated to occur in the hysteresis loops associated with the higher field regions. In the fluid, the interaction between individual SAF structures in a magnetic field is a complicated one. The SAF will couple with dipole-dipole (d-d) energy.

The form of the $d-d$ coupling is included into the effective field Eq. (3) of the LLG Eq. (2) and is given by

$$
E_{\mathrm{d}-\mathrm{d}}=\frac{\mu_{0} V_{\mathrm{A}} V_{\mathrm{B}}}{r^{3}}\left[\mathbf{M}_{\mathrm{A}} \cdot \mathbf{M}_{\mathrm{B}}-3\left(\mathbf{M}_{\mathrm{A}} \cdot \frac{\mathbf{r}}{r}\right)\left(\mathbf{M}_{\mathrm{B}} \cdot \frac{\mathbf{r}}{r}\right)\right]
$$

where $\mathbf{M}_{\mathrm{A}}=\left\langle\mathbf{M}_{1}+\mathbf{M}_{2}\right\rangle$ and $\mathbf{M}_{\mathrm{B}}=\left\langle\mathbf{M}_{3}+\mathbf{M}_{4}\right\rangle$ are for two coupled SAF. The nanostructures move in a spherical coordinate system, as is shown in Fig. 3. We find that in a chain of the SAF the saturation field is reduced when the nano-magnetic structures are in close enough proximity for the dipole-dipole energy to be close to that of the coupling energies between SAF nanomagnets, i.e.,

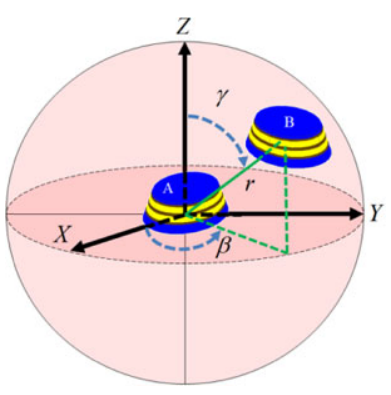

Figure 3 The interaction of two SAF structures, A and B, in a spherical coordinate system.

$\left|\mu_{0} V_{\mathrm{A}} V_{\mathrm{B}} / r^{3}\right| \rightarrow\left|\mu_{0} V J\right|$. Due to the offset of the easy axes of the SAF, there is a continual rotation and this creates a fluctuation in the $d-d$ energy because there are deviations in the separating distance and relative orientations. As a result, the hysteresis of the system changes in accordance with the closeness of the SAF and their interaction. When there is no applied magnetic field, the mean value of the magnetization is zero and as such that there is no remanence or coercivity observed. In Fig. 4, we show the results of our simulations against the experimentally found results of Koh et al. [2]. The nanomagnets structures have larger energy losses compared to before they are released into solution due to their motion and proximity. The continual motion of the particles can result in shifts in the hysteresis profile. In Fig. $4 \mathrm{a}$ and $\mathrm{b}$, there are two slightly differing hysteresis pathways that can be found due to fluctuations in their position.
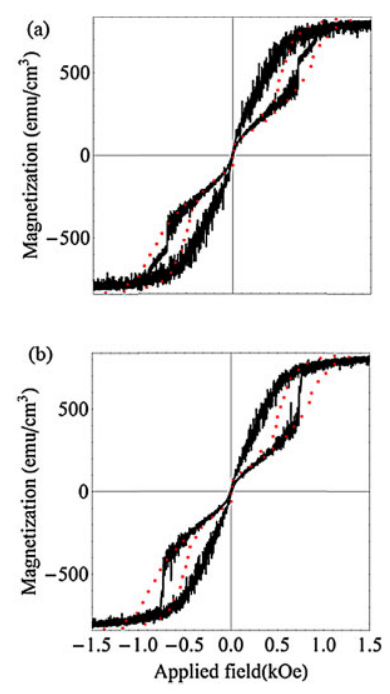

Figure 4 After the ion milled SAF are released into fluid, their hysteresis changes so that there is a greater energy loss and lower saturation field. The dipole-dipole interaction between the SAF structures is mostly responsible for this change. In (a) and (b) for the same system of coupled SAF, slightly altered hysteresis profiles are seen due to random fluctuations in their relative position with regards to each other. The experimental values of Koh et al. [2] are shown by red dots. 
If the SAF are deliberately fabricated as elliptical cones from the beginning then the easy-axis should remain fixed after ion-milling. In this scenario, the system will be more robust against energy losses.

2.2 Nano-mechanics and hysteresis In Fig. 2, the hysteresis after ion milling is shown. The size of the structure is diminished in the process [2], including the average thickness of the magnetic layers. As this is a dynamical study, we also include an indication of the switching times of the magnetic moments of the structures (see Ref. [8] for a detailed analysis of switching in two magnetic layer systems). In Fig. 2c, the average magnetization, $\approx \partial E / \partial H$, is shown as it evolves in time. The switching from $+M_{\mathrm{S}}$ to $-M_{\mathrm{S}}$ occurs in about $2 \mathrm{~ns}$. The components of $\mathbf{M}_{1 / 2}$ as a function of time can be seen in Fig. 2d-f. The magnetic moments are mostly constricted to move in the $x-y$ planes of the nanomagnets. Figures $1 \mathrm{a}$ and 2a both have extra "spikes" in the hysteresis profiles just before the saturation fields, that are not seen in the

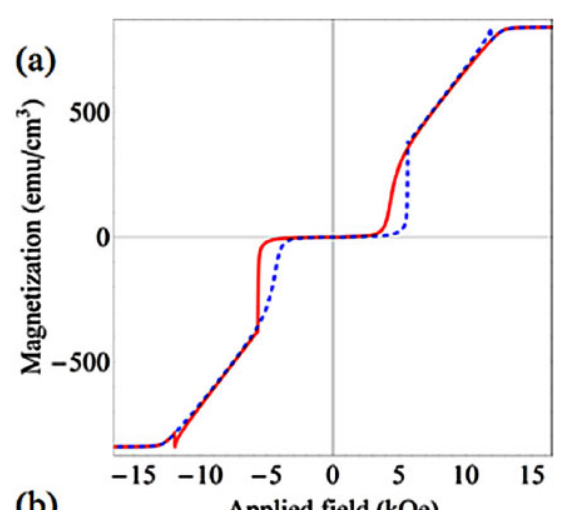

(b)

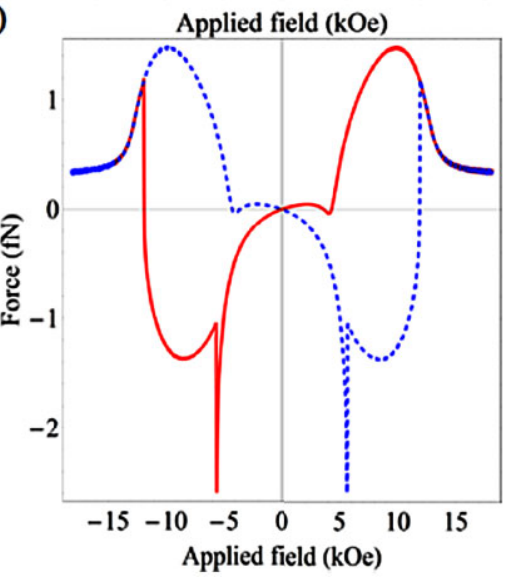

Figure 5 The magnetization (a), and magnetic forces (b), of a SAF structure $\mathrm{Co}_{90} \mathrm{Fe}_{10}(2) / \mathrm{Ru}(2) / \mathrm{Co}_{90} \mathrm{Fe}_{10}(5)$ in an applied magnetic field. The bottom nanomagnet has dimensions, $l_{x}=50 \mathrm{~nm}$ and $l_{y}=30 \mathrm{~nm}$, whereas for the top one $l_{x}=40 \mathrm{~nm}$ and $l_{y}=20 \mathrm{~nm}$. The red solid line (dashed blue line) indicates the hysteresis path between parallel alignments of the magnetic moments in the two nanomagnets as the magnetic field amplitude goes from a positive (negative) to a negative (positive) value. experimental data. This may be because of the size of the field increments used in the experiments, which are typically around $50 \mathrm{Oe}$, and these small spikes may have been missed.

Next, we focus on elliptical nano-magnetic elements, the magnetic response of the nanostructure to an applied magnetic field and the resulting magnetic forces. These magnetic fields can induce mechanical motion of SAF structures when they are immersed in a fluid. For a number of medical applications, SAF nanoparticles with dimensions smaller than $50 \mathrm{~nm}$ are desirable. Thus, a SAF structure of elliptical proportions smaller than this has been analyzed and the results are shown in Fig. 5.

Any mechanical motion of nanoparticles must have an effect on the fluctuating cellular environment and so it is important to gauge the level of force attributable to magnetic motion alone. This will give us a more intuitive feel for how the SAF structures could be controlled magnetically in vivo and how their contact with the biological cells may affect the biological environment. The coordinated functioning of cells, and their manipulation, is largely dependent upon their responses to varying levels of mechanical signaling and stimulation. The cells of the body are regulated by signal-transduction mechanisms that result in force induced chemical triggers that give rise to signaling cascades. The opening of mechano-transduction pathways results in the interior of the cell structurally changing and consequently deforming the shape and mechanical stability of the nucleus [9]. This brings into question the safety of delivering high doses of nanoparticles without understanding how their characteristics change in vivo and their consequent effects on the body when interacting with cells and tissues. The nanoparticles have to be designed to evade or interact beneficially with the immune system [10] in order to reach their target within the body and not interfere with biological cells that function as part of the active immune system that is combating a targeted disease. The incorporation of magnetic nanoparticles into therapeutic and drug delivery systems [11] must take these factors into account as toxicological or inflammatory damage to cells can occur through mechanical motion and heating [12]. The responsiveness of the nanomagnets to applied magnetic fields should be known and the relaxation in the applied field analyzed to maximize efficacy.

For a preferential encounter between the SAF structures and the immune system, the correct magnetic field strengths and magnetic forces should be applied in accordance with the objective of the interaction, e.g., piconewton forces applied to cancer cells to initiate apoptosis with minimal necrotic side-effects. The incorrect magnetic fields could incur the development of exaggerated reactions from the immune system, detrimental to recovery and with the heightened possibility of autoimmune and chronic inflammatory diseases emerging.

Figure 5 shows the hysteresis profile with a typical spinflop (e.g., $[8,13,14])$ appearance for an independent SAF structure. Here, we deliberately sought to give an example whereby there are clear hysteresis losses. The generic 
magnetic force for a multilayered magnetic structure is given by

$$
F_{\mathrm{SAF}}=2 \mu_{0} \mu_{\mathrm{r}} \sum_{i}^{N} H_{\mathrm{a}} M_{s, i} V_{i} \frac{\sin \left(\beta-\varphi_{i}\right)}{l_{x, i}} .
$$

The angle at which the field, $H_{\mathrm{a}}$, is applied is $\beta$ and in the discussed case the number of nanomagnets in the SAF is $N=2$. The relative magnetic permeability $\left(\mu_{\mathrm{r}}\right)$ of the SAF structure, as well as the number of magnetic elements, can greatly change the force generated. The magnetic permeability is a function of the frequency of the applied magnetic field and for CoFe structures is typically about $\mu_{\mathrm{r}}=100$ at the considered frequency of $1 \mathrm{GHz}$ [15]. Changing the magnetic material to higher permeability substances such as permalloy, which can have permeability 2 orders of magnitude higher than soft iron, can radically change the force distribution and the applicability of the SAF structure. Here we show femtonewton force distributions that peak when there is a Barkhausen jump [16] from one magnetic state into another (e.g., scissored magnetic moments to anti-parallel). Identifying where these peaks in magnetic force occur is important because there might be times in operating a functional set of SAF structures where that extra nudge is required. Also, by identifying at which magnetic field strengths small hysteresis loops occur one is able to introduce a static magnetic field to the system to bias it towards a chosen loop, as is shown in Fig. 6.

In Fig. 6, the system has been biased to operate only in the local vicinity of one of the small hysteresis loops that can be seen in Fig. 5. Thus, we demonstrate that by applying a static magnetic field half way between the maximum and minimum field strengths for this small loop of Fig. 5 that one can then reduce the amplitude of an applied AC field so as to operate the system with magnetization changes in respect to this loop exclusively. After the initial switching on of the AC field, the system then finds a secondary smaller hysteresis cycle that operates continuously (green and blue lines in Fig. 6). The resulting magnetic force is shown in Fig. 6b. In Fig. $6 \mathrm{c}$ and $\mathrm{d}$, the switching of the magnetization state of each nanomagnet in the SAF structure is plotted. Therewith, the magnetizations of the two magnets are seen to never move in parallel. The secondary smaller hysteresis loop can be expanded by increasing the frequency of the AC field. Indeed, this can be seen for the same SAF set-up as demonstrated in Figs. 5 and $6 a-d$ but with a frequency of $50 \mathrm{GHz}$ in Fig. 6e. Thus, we can control the nature of the SAF structure by building it with a material of choice to define the relative permeability and by changing the frequency applied, as well as geometric rearrangement. Combining these principles for the design of SAF structures and the previously developed fabrication techniques [2] will allow one to impose an unprecedented range of forces, from a few femtonewtons to a few hundred piconewtons on a cellular membrane. The mechanical characterization of individual and groups of SAF in magnetic fields is important for future cellular behavior and physiological function (a)

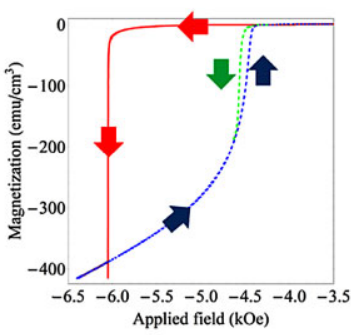

(b)

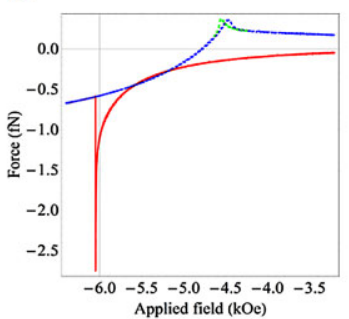

(c)

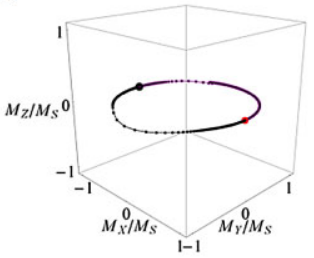

(d)

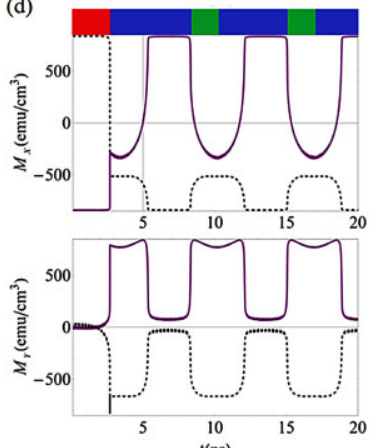

(e)

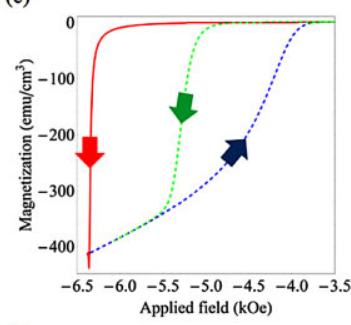

(f)

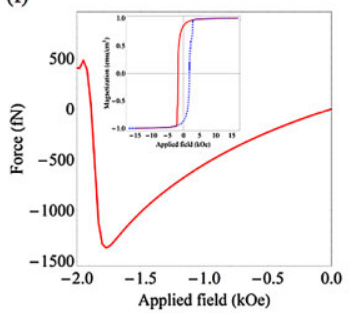

Figure 6 A static magnetic field is applied along the longest axis of the SAF, $H_{\text {static }}=-5.25 \mathrm{kOe}$, and an oscillating field with amplitude $H_{\text {applied }}=-1.75 \mathrm{kOe}$ is also applied at an angle of $\beta=7^{\circ}$. The nanomagnet has dimensions as in Fig. 5. In (a), the hysteresis loop that is maintained in the vicinity of $H_{\text {static }}$ is shown. (b) The magnetic force of the SAF structure as the AC field changes. (c) The magnetic moments are shown to be largely constrained to move in the $x-y$ plane, as shown in this $M_{x / y / z}$ plot. In (d), the time evolution of the $M_{x}$ and $M_{y}$ components of the magnetization are shown for each nanomagnet (purple solid lines for the first magnet and dashed black lines for the second). The red solid lines represent when the AC field is turned on and the field is in reverse. The blue dashed lines are the forward part of the AC field cycle. Subsequent reversals of the field give rise to magnetizations and forces shown by green coloration and dashed lines. (e) Hysteresis paths for the same SAF structure as in $(a-d)$, except the frequency of the magnetic field has been increased to $50 \mathrm{GHz}$. (f) The magnetic force for a SAF structure with three permalloy nanomagnets with dimensions $l_{x}=150 \mathrm{~nm}, l_{y}=50 \mathrm{~nm}$, and $l_{z}=10 \mathrm{~nm}$. The effective magnetic permeability is $\mu_{\mathrm{r}}=1000$ at a frequency of $1 \mathrm{GHz}$.

analyses. The problems associated with the calibration of superparamagnetic beads to exert small forces (that are due to irregular magnetic susceptibilities $[1,2]$ ) are overcome by the relative heterogeneity of the sizes of SAF elements designed as elliptical cones.

3 Conclusions We have taken the SAF structures that consist of two coupled nanomagnets that are similar to those 
fabricated by Koh et al. [2, 16] and shown that the magnetically induced forces, and hence possible stresses, are not constant but vary as a function of the applied field. In fact, the critical fields at which point the magnetic moments suddenly change orientation result in the generation of the maximal forces. We have considered non-ionizing magnetic fields applied at radio frequencies to the SAF structures. The magnetic response is modified by geometrical changes incurred by ion milling. These results were obtained by solution of the LLG equations for SAF structures where the magnetic elements have misaligned orientations. We have found the switching times of the SAFs from magnetic to nonmagnetic states. This finding can optimize the duration of applying a magnetic field in techniques such as cancer signal-transduction therapy. From this, one can then apply the correct magnetic field amplitude and frequency in short pulses so as to minimize magnetically incurred heating when carrying out mechanical therapy on cellular structures. We have shown that the magnetic forces originating from the SAF can be tuned by the choice of material for the magnetic elements, the applied frequency and the geometry of the structure. The nano-mechanical stimulation of biological entities with nanomagnets, based upon these principles, is indicated as a future technique to understand their complex behavior and workings. Using short duration magnetic pulses and a rotating magnetic field will allow one to maintain the applied force sufficiently for the SAF structures to rotate like a compass needle in solution. Dynamical analysis of the processes and the interaction of molecules is a growing area of research [17-19] and the enhanced information about SAF structures discussed herein will assist in the next functional investigation of bio-molecules by the creation of variable strength magnetic torque synthetic nanomaterials.

Acknowledgements M.F. thanks the EPSRC for funding under KTA grant - "Developing prototypes and a commercial strategy for nanoblade technology."

\section{References}

[1] W. Hu, R. J. Wilson, A. L. Koh, A. Fu, A. Z. Faranesh, C. M. Earhart, S. J. Osterfeld, S.-J. Han, L. Xu, S. Guccione, R. Sinclair, and S. X. Wang, Adv. Mater. 20, 1479 (2008).

[2] A. L. Koh, W. Hu, R. J. Wilson, C. M. Earhart, S. X. Wang, and R. Sinclair, J. Appl. Phys. 107, 09B522 (2010).

[3] Y.-F. Chen, J. N. Milstein, and J.-C. Meiners, Phys. Rev. Lett. 104, 048301 (2010).

[4] J.-Y. Shao, Ann. Biomed. Eng. 30, 546 (2002).

[5] D.-H. Kim, E. A. Rozhkova, I. V. Ulasov, S. D. Bader, T. Rajh, M. S. Lesniak, and V. Novosad, Nature Mater. 9, 165-171 (2010).

[6] D. M. Forrester, K. E. Kürten, and F. V. Kusmartsev, Phys. Rev. B 76, 134404 (2007).

[7] M. Beleggia, M. De Graef, Y. T. Millev, D. A. Goode, and G. Rowlands, J. Phys. D: Appl. Phys. 38, 3333 (2005).

[8] M. Forrester, F. Kusmartsev, and E. Kovács, Phys. Rev. B 87, 174416 (2013).

[9] J. N. Milstein and J.-C. Meiners, J. R. Soc. Interface 8, 1673 (2011).

[10] A. L. van de Ven, P. Kim, O. Haley, J. R. Fakhoury, G. Adriani, J. Schmulen, P. Moloney, F. Hussain, M. Ferrari, X. Liu, S. H. Yun, and P. Decuzzi, J. Control. Release 158, 148 (2012).

[11] A. M. Derfus, W. C. W. Chan, and S. N. Bhatia, Adv. Mater. 16, 961 (2004).

[12] A. O. Govorov and H. H. Richardson, Nano Today 2(1), 30 (2007).

[13] B. Negulescu, D. Lacour, M. Hehn, A. Gerken, J. Paul, and C. Duret, J. Appl. Phys. 109, 103911 (2011).

[14] D. C. Worldege, Appl. Phys. Lett. 84(15), 2847 (2004).

[15] H. S. Jung and W. D. Doyle, IEEE Trans. Magn. 38, 2015 (2002).

[16] A. L. Koh, W. Hu, R. J. Wilson, S. X. Wang, and R. Sinclair, Philos. Magn. 88(36), 4225 (2008).

[17] H. Clausen-Schaumann, M. Seitz, R. Krautbauer, and H. E. Gaub, Curr. Opin. Chem. Biol. 4, 524 (2000).

[18] I. Frank and J. Friedrichs, Nature Chem. 1, 264 (2009).

[19] 1. V. Pobelov, G. Mészáros, K. Yoshida, A. Mishchenko, M. Gulcur, M. R. Bryce, and T. Wandlowski, J. Phys.: Condens. Matter 24, 164210 (2012). 